\title{
Distortions and deletions: Word-initial consonant specificity in fluent speech
}

\author{
LARRY H. SMALL \\ Bowling Green State University, Bowling Green, Ohio \\ and
}

Z. S. BOND

Ohio University, Athens, Ohio

\begin{abstract}
This study investigated the perceptual effects of either distorting (misarticulating) or deleting word-initial phoneme targets located in two-syllable words. Subjects were instructed to shadow taped prose passages containing the distorted or deleted targets. The subjects typically repeated words containing distorted targets as heard on the tape, and they typically restored words containing deleted targets. That is, the subjects reconstructed the missing acoustic-phonetic information even with deletions as large as $137.5 \mathrm{msec}$. Deleting word-initial consonants apparently had little effect on the word-recognition process during shadowing. The subjects appeared to rely on contextual constraints in lieu of the missing acoustic-phonetic information.
\end{abstract}

The experimental study of fluent speech perception dates back to at least 1900 with the publication of William Chandler Bagley's (1900-1901) paper, "The Apperception of the Spoken Sentence: A Study in the Psychology of Language."' In his paper, Bagley examined the role of lexical and semantic constraints in fluent speech perception.

Since Bagley's time, much research in fluent speech perception has centered on recognizing words in noisy environments. The majority of the literature has stressed the importance of lexical, semantic, and prosodic constraints provided in spoken utterances which help listeners to recognize words in noisy situations.

Some of this research has dealt with what is now called the "phonemic restoration effect" (Warren, 1970). This auditory illusion, first demonstrated by Bagley, involves a listener's ability to restore missing acoustic information due either to a distorted speech signal or to noise. More recently, the role of phonemic restorations in word recognition in fluent speech has been described by Samuel (1981).

Although much research has dealt with redundancies in the spoken message, less effort has been given to the study of perception of segments, even though the auditory cues for segment identity present in isolation are often different from those observed in fluent speech (Cole \& Jakimik, 1980). Studies involving segmental perception have largely examined the role of consonant distinctive features in word recognition (Cole, 1973; Marslen-Wilson \& Welsh, 1978).

\footnotetext{
A preliminary version of this paper was presented at the Spring meeting of the Acoustical Society of America, Austin, Texas 1985. L. H. Small's mailing address is: Communication Sciences and Disorders, 337 South Hall, Bowling Green State University, Bowling Green, OH 43403.
}

Cole (1973) utilized a misarticulation detection task in which phonemes were altered by one, two, or four distinctive features using the Keyser and Halle (1968) feature system. Subjects detected only $30 \%$ of the one-feature mispronunciations. As the number of distinctive feature changes increased, so did the subjects' detection rates; two and four feature changes were detected with $60 \%$ and $75 \%$ accuracy, respectively.

Utilizing a shadowing task, Marslen-Wilson and Welsh (1978) examined subjects' responses to misarticulated three-syllable words. The misarticulations involved altering a phoneme by one or three distinctive features (Keyser \& Halle, 1968, system) in either the first or third syllable. Marslen-Wilson and Welsh discovered that subjects fluently restored one-feature misarticulations with the greatest proficiency in the third syllable in a highly constrained context.

These studies demonstrated that as the number of distinctive feature changes increases, the identity of the target phoneme becomes less apparent. In detection tasks, this is evidenced by an increase in the number of detections, as the number of feature changes increases. In shadowing tasks, the greater the number of feature changes, the fewer the fluent restorations. Presumably, if a feature change is such that it has little effect on a subject's perception of a target (that is, a miss in a detection task or a fluent restoration in a shadowing task), that feature is not critical in perception of a particular target. For example, voicing changes apparently have little effect in fluent speech perception since their restoration rate in shadowing is quite high (Bond \& Small, 1983).

The first purpose of the present study was to examine subjects' ability to shadow, in continuous speech, words that were altered in their distinctive feature specifications. Subjects shadowed words that were altered by various 
one- and three-distinctive-feature changes so that the individual effect of a feature change on a listener's ability to perceive fluent speech could be examined.

The second purpose of the present study was to investigate the effect of completely deleting consonants from the spoken message in order to determine whether a phonemic restoration effect could be demonstrated for deleted consonants in a shadowing task. Although this illusion has been demonstrated in other experimental tasks, it has not, to our knowledge, been demonstrated in shadowing fluent speech.

If, indeed, words can be recognized without complete acoustic specifications (that is, with missing initial consonants), the cohort theory of word recognition as originally stated (Marslen-Wilson \& Welsh, 1978) will be put into question. The cohort theory states that word recognition occurs after the initial speech segment of a word is heard and a pool of word candidates is activated. This initial speech segment was originally believed to consist solely of the word-initial phoneme (Marslen-Wilson \& Welsh, 1978). More recently, the initial segment necessary for the activation of a pool of word candidates has been believed to be of syllabic length, that is, that it contains the first two word-initial phonemes of a particular word (Salasoo \& Pisoni, 1985). Both Salasoo and Pisoni (1985) and Tyler (1984) have recently shown that the initial segment necessary for activating a pool of word candidates is more on the magnitude of $150 \mathrm{msec}$ long.

\section{METHOD}

\section{Speaker}

An experienced male speaker, with a theatrical background, recorded all of the experimental tapes and the practice tape at a rate of approximately $160-170$ words $/ \mathrm{min}$. The speaker produced all of the alterations or mispronunciations on the first two experimental tapes after considerable practice with the material. All recordings were made in a double-walled, sound-treated room (IAC No. 402). The speaker wore a Sony electret condensor lavalierstyle microphone (ECM-150), coupled to a Pioneer RT-707 reelto-reel recorder for all recordings.

\section{Materials}

The test materials consisted of recordings of prose passages taken from a novel with a simple prose style. The experimental passage was 635 words in length and included 14 two-syllable test words which were altered for the three experimental conditions. A second simple prose passage of approximately the same length was selected for the recording of a practice tape. No alterations occurred on the practice tape.

All the test words from the experimental passages were selected to be highly predictable from context. To determine predictability, a group of 10 undergraduate and graduate students were used as subjects. These 10 șubjects were given 80 written sentences taken from the experimental prose passage. Each sentence had one twosyllable word omitted. The subjects simply filled in the blank with the word they deemed most appropriate in each sentence. Predictability from context, or contextual constraint, for each word was determined by using a scoring scheme based on Marslen-Wilson and Welsh (1978). If the subject's written response was the omitted word, it was scored " 1 "; a synonym was scored " 2 "; a related word was scored " 3 "; and an unrelated word was scored "4." Fourteen test words were selected from the 30 words with the lowest mean rating (most predictable from context), on the basis of the following phonetic-structure and lexical-stress requirements: (1) Lexical stress to be on the first syllable of each two-syllable test word. (2) Each initial phoneme to be either an obstruent or the approximants $/ \mathrm{r} /$ or $/ /$ (the use of the other English phonemes would not allow the appropriate distinctive feature changes needed for the experimental conditions). (3) Target phonemes to be composed of as many varied initial phonemes as possible that met requirement 2 . (4) All one-feature changes to occur within each comparable threefeature change.

The mean contextual rating for the 14 selected test words was $2.08(S D=0.38 ;$ range $=1.5-2.5)$. According to the Kucera and Francis (1967) norms, the mean frequency of occurrence in English for the 14 words was $85.93 /$ million $(S D=107.57$; range $=$ 1-393).

The 14 test words were altered in one of three ways to construct three experimental tapes. On the first experimental tape, the onefeature condition, the initial phoneme in each test word was changed by one distinctive feature, for example, "seated" was changed to /fired/ (+coronal to -coronal change). The one-distinctive-feature changes used in this study included coronal, strident, voicing, continuant, and anterior changes. (See Table 1 for the specific feature changes used in this condition.) These features were chosen because they were the only one-feature changes possible after test word selection, as guided by the four requirements previously mentioned.

On the second experimental tape, the three-feature condition, the initial phonemes of the 14 test words were changed by three features, for example, "seated" was changed to /pirəd/ (tcoronal to -coronal, +continuant to -continuant, +strident to -strident). Based on the phonetic identity of the initial 14 consonants, there were also a limited number of possible three-feature changes. The specific changes used are given in Table 2, using the Chomsky and Halle (1968) feature system.

On the third experimental tape, the deletion condition, the initial phoneme of each test word was deleted completely, for example, "seated" was changed to /irəd/. This was accomplished by mechanically removing the initial phoneme of the test word, instead of having the word spoken with the sound omitted, in order to maintain the proper timing of the word in the sentence. A recording of the prose passage (at 13.5 ips) with no alterations on it, was pulled, by hand, over the heads of a reel-to-reel recorder (Pioneer RT-707), so that the initial phoneme of each of the 14 test words could be identified on the tape. To "erase" the initial phoneme, the tape was removed from the recorder and a section of the magnetic backing was eradicated with a cotton swab and acetone. After a section of tape was eradicated, the tape was played normally on the Pioneer

Table 1

Types of One-Feature Changes for the 14 Test Words in the One-Fenture Condition

Test Word Voicing Coronal Continuant Anterior Strident

(Phoneme Change)

seated $(f)$

vanished (f)

ceiling $(\theta)$

figures (s)

tutor (p)

rancid (1)

silence $(z)$

person (t)

city (f)

touching (d)

building $(p)$

daughter (o)

satin $(\theta)$

ready (1)
$+1-$

$-1+$
$+1-$

$-1+$

$-1+$

$-1+$

$+1-$

$+1-$

$-1+$

$+1-$

$-1+$

Note-Distinctive feature changes: + to $-(+/-)$ or - to $+(-/+)$. 
Table 2

Type of Three-Feature Changes for the 14 Test Words in the Three-Feature Condition

\begin{tabular}{|c|c|c|c|c|c|c|c|c|}
\hline Test Word & Voicing & Coronal & Strident & Continuant & High & Anterior & Sonorant & Vocalic \\
\hline (Phoneme Chan & & & & & & & & \\
\hline $\begin{array}{l}\text { seated (p) } \\
\text { vanished }(\theta) \\
\text { ceiling }(d) \\
\text { figures }(D) \\
\text { tutor }(f) \\
\text { rancid }(\delta) \\
\text { silence (d) } \\
\text { person (s) } \\
\text { city (p) } \\
\text { touching (z) } \\
\text { building }(\theta) \\
\text { daughter (1) } \\
\text { satin }(d) \\
\text { ready }(\delta)\end{array}$ & $\begin{array}{l}-1+ \\
-1+ \\
+1- \\
-1+\end{array}$ & $\begin{array}{l}+1- \\
-1+ \\
-1+ \\
+1- \\
-1+ \\
+1- \\
-1+\end{array}$ & $\begin{array}{l}+1- \\
+1- \\
+1- \\
-1+ \\
+1- \\
-1+ \\
+1- \\
-1+ \\
+1-\end{array}$ & $\begin{array}{l}+1- \\
+1- \\
-1+ \\
+1- \\
-1+ \\
+1- \\
-1+ \\
-1+ \\
-1+ \\
+1-\end{array}$ & $-1+$ & $\begin{array}{l}+1- \\
-1+\end{array}$ & $\begin{array}{l}-1+ \\
+1-\end{array}$ & $-1+$ \\
\hline
\end{tabular}

recorder to determine, by listening, how much of the phoneme had actually been removed. When it appeared that the phoneme had been completely eradicated, the test word was rerecorded from the $\mathrm{Pi}$ oneer recorder to a second reel-to-reel recorder (Dokorder No. 4000 ).

The criteria for determining how much magnetic backing should be removed were based solely on listening judgments. Once the experimental tape was completed, it was judged by one experimenter (L.S.) and by two other experienced listeners. If there were any disagreements, the words were reconstructed until there was $100 \%$ agreement among the three listeners that the first phoneme had been completely eradicated.

The experimental tape with the 14 completed test words was analyzed spectrographically (Voice Identification, 700 series). To determine exactly how much of the word had been eradicated, a spectrogram of the test word with the phoneme deleted was compared with a spectrogram of the same word unaltered. In most cases, it was impossible to remove only the initial phoneme in question without removing a portion of the preceding or following phonemes, since, as is well known, phonemes in words are not produced in serial order, but in parallel (Liberman, Cooper, Shankweiler, \& Studdert-Kennedy, 1967).

Each of the spectrograms of the test words was measured to determine the amount of speech signal eradicated in each case (in milliseconds) and how much of the preceding or following phoneme had to be removed. To determine segmental boundaries, the criteria reported by Peterson and Lehiste (1960) were adopted for this study. The deleted portion of each target word is indicated in Table 3 .

\section{Subjects}

Forty-eight native English-speaking students at Ohio University with no hearing or speech problems served as subjects. These students were undergraduates enrolled in classes in the School of Hearing and Speech Sciences, and they participated in the experiment in partial fulfillment of course requirements. Sixteen subjects ( 8 males, 8 females) were assigned to each of the three experimental conditions, so that each subject received only one of the tests.

\section{Procedure}

All subjects were told that they would be listening to two passages of a story. They were instructed to repeat, as rapidly as possible, everything they heard the speaker say. The instructions stressed repetition, rather than paraphrasing, of the passages. Each subject received the practice tape first and then the experimental tape. Before the subject heard the experimental tape, he or she was told that some "strange" things would be heard in the taped passage, but that he or she should still repeat everything that was heard, exactly as spoken. This was done to promote accurate shadowing, so that any restorations that occurred would not be due to the subject's consciously supplying the restorations.

Table 3

Proportion of Words Deleted (in Milliseconds) for the Deletion Condition

\begin{tabular}{|c|c|c|}
\hline Word & Total Removed & Phonemes Deleted \\
\hline $\begin{array}{l}\text { seated } \\
\text { ready } \\
\text { vanished } \\
\text { tutor } \\
\text { daughter } \\
\text { touching } \\
\text { (the) city } \\
\text { building } \\
\text { person } \\
\text { (smelled) rancid } \\
\text { (the) ceiling } \\
\text { figures } \\
\text { (blue) satin } \\
\text { silence }\end{array}$ & $\begin{array}{r}106.25 \\
87.50 \\
103.13 \\
59.38 \\
56.25 \\
50.00 \\
93.75 \\
40.63 \\
56.25 \\
90.63 \\
87.50 \\
81.25 \\
115.63 \\
137.50\end{array}$ & 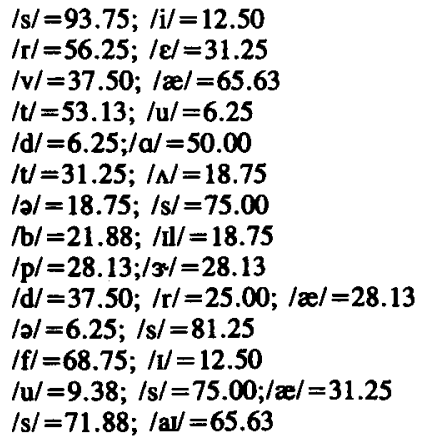 \\
\hline \multicolumn{3}{|c|}{ Mean $=83.26$} \\
\hline
\end{tabular}


Subjects who failed to learn the shaciowing task during the practice session were excluded from the study, as were subjects who were subjectively considered to be poor shadowers, that is, those who could not shadow within two or three words of the taped passage. This resulted in the exclusion of 5 subjects from the study; they were replaced by 5 other subjects.

To make sure that the subjects had comprehended the passages they had heard, they were asked, at the end of both the practice and the experimental tapes, to orally answer five questions. Failure to correctly answer three or more of the questions concerning the experimental passage also resulted in exclusion from the study. Four subjects were excluded for this reason (with replacement).

The subjects were tested in an IAC No. 402 double-walled, soundtreated room; they listened to the tapes at a presentation level of $70 \mathrm{~dB}$ SPL through headphones (Grason-Stadler TDH 39). The tapes were played on a Pioneer reel-to-reel tape recorder (RT-707) with a Sansui integrated amplifier (A-40). The signal delivered to the headphones was binaural.

One channel of the Pioneer recorder was connected to one channel of a Dokorder (No. 4000) four-channel reel-to-reel recorder. Each subject wore a Sony electret condensor lavalier-style microphone (ECM-150) coupled to the second channel of the Dokorder recorder. Each subject's responses, as well as the spoken experimental passages, were recorded simultaneously for later analysis.

\section{RESULTS}

\section{Classification of Responses}

Subjects' responses to the test words when they were shadowing were grouped into three major categories: restorations, defined as corrections of the altered stimuli, repetitions, and omissions. Restorations were further subdivided into two types: (1) complete restorations-the subject corrected the altered word to its original form-and (2) partial meaningful restorations-the subject substituted a real word for the altered word, but not the word in the original passage. In both cases, subjects arrived at a meaningful lexical item.
Repetitions were defined as a repetition of the test word as altered on the tape. Also counted as repetitions were meaningless phonetic sequences in place of the altered word. Omissions included both omissions of the altered word and completely unintelligible responses.

The shadowed responses of the targets for all subjects for the three experimental conditions are shown in Figure 1 . It can be seen that for the one-feature- and threefeature-change conditions, subjects tended to repeat the mispronounced target-bearing words, whereas, in the deletion condition, they tended to restore the mispronounced words to their original forms. With restorations, subjects in the one-feature-change condition completely restored target-bearing words only $33 \%$ of the time and showed partial meaningful restorations (i.e., meaningful newword responses) $7 \%$ of the time. In the three-feature condition, subjects' responses showed $16 \%$ complete restorations and $4 \%$ partial meaningful restorations. However, in the deletion condition, a total of $76 \%$ of the subjects' responses were restorations, $57 \%$ complete and $19 \%$ partial meaningful.

In relation to repetitions in the one-feature-change condition, $49 \%$ of the subjects' responses were exact repetitions and only $2 \%$ of the responses were meaningless phonetic sequences (for a total of $51 \%$ ). Likewise, in the three-feature-change condition, subjects showed a total $68 \%$ repetition rate, with meaningless phonetic sequences accounting for $13 \%$ of the responses. In the deletion condition, the repetition rate was quite low, both for exact repetitions (6\%) and for meaningless phonetic sequences (7\%).

Since complete restorations were of specific interest, only this response type was examined in a three-factor analysis of variance (ANOVA). The ANOVA showed a significant main effect for differences between conditions
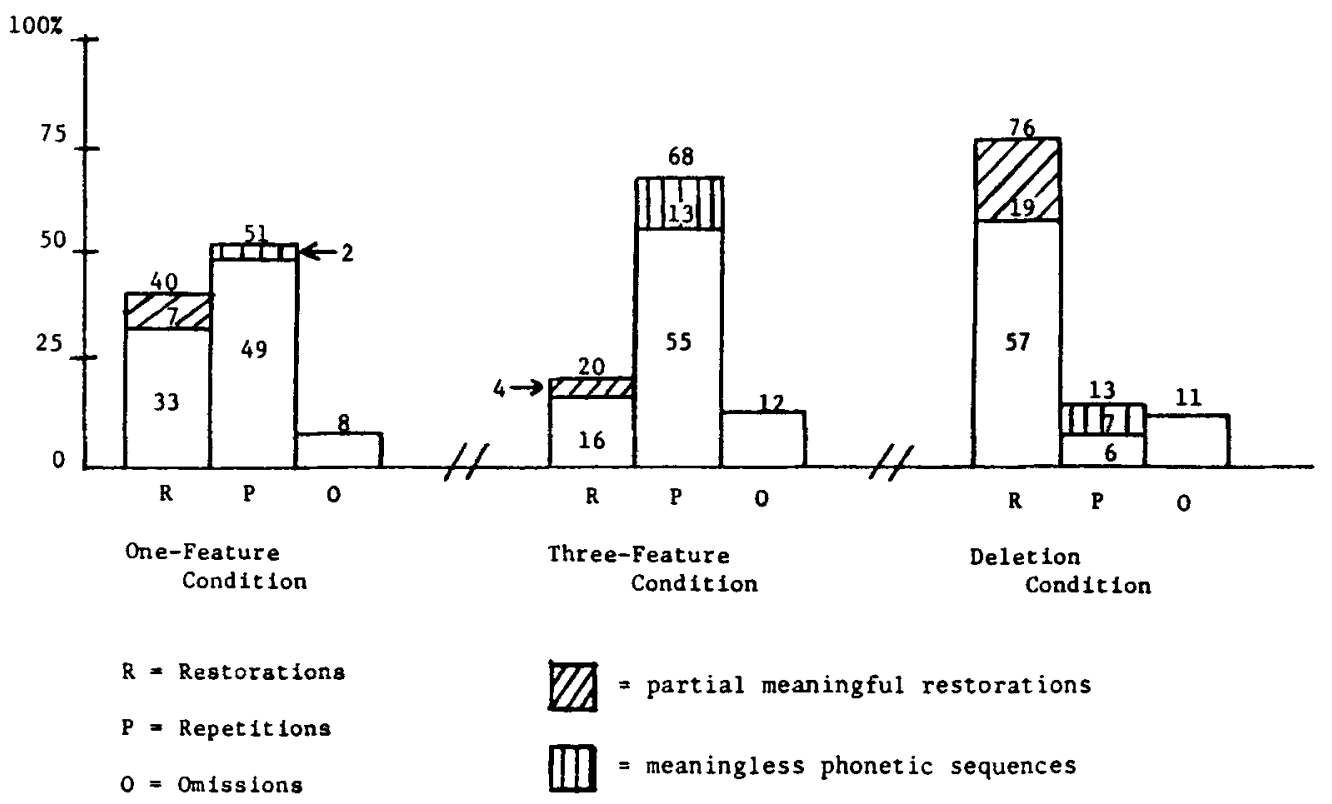

Figure 1. Percentages of subjects' response types for the three experimental conditions. 
$\left[\min F^{\prime}(2,28)=10.14, p<.001\right]$ (Clark, 1973). A posteriori tests on condition showed significant differences between the deletion condition and the one-feature condition, and between the deletion condition and the three-feature condition; no difference was found between the one- and three-feature-change conditions [Tukey HSD, $p<.05$ ] (Bruning \& Kintz, 1977).

Although altering words by either one or three features appeared to make no difference in subjects' responses to these misarticulations, there was a difference between whether the word-initial consonant was misarticulated (or distorted) or was completely deleted. The subjects tended to restore missing phonemes much more readily than they did word-initial phonemes that were distorted.

The ANOVA showed no significant main effect for differences between test words in each condition. The design of this ANOVA was not sensitive to actual changes in distinctive features because the features employed could not be grouped as a separate variable. The ANOVA was also not sensitive to differences between words in the deletion condition.

The ANOVA also indicated no significance for the main effect of sex. As indicated by the number of restoration responses seen, the shadowing task appeared to be one that could be handled similarly by both sexes.

No significant double or triple interactions were observed among any of the main effects.

The subjects' mode of responding to a deleted wordinitial consonant appeared to be quite different from that for a distorted word-initial consonant. Since the restoration data from the two distinctive-feature-change conditions were statistically similar, the data from these two conditions will be referred to collectively as the "distortion conditions."

\section{DISCUSSION}

The results of this study indicated that subjects' shadowed responses differed for words that were altered by misarticulation of the initial consonant and those that were altered by its complete deletion. When a word-initial consonant was completely missing, listeners appeared to have little trouble in restoring the missing sound. Therefore, the phonemic restoration effect was demonstrated by subjects when shadowing fluent speech. However, when the word-initial consonant was misarticulated, by either one or three distinctive features, the subjects had more difficulty in restoring the targets to their original forms. Instead, the subjects tended to repeat the misarticulated phonemes.

\section{Distortions versus Deletions}

In examining why deletions were restored more often than distortions in word-initial consonants, it must be remembered exactly what was being manipulated in each condition. In the case of deletions, listeners were given information that was phonetically impoverished, whereas in the case of distortions, listeners were hearing nonwords with complete acoustic specifications, although the acoustic cues did not cue perception of meaningful words. That is, in the case of distortions, the acoustic cues alerted listeners to the fact that a word had occurred, but the word was not located in their mental lexicons. In the deletion condition, listeners must have relied quite heavily on the context of the passage in order to make some sense out of target-bearing nonwords with deleted initial phonemes. This was reflected in the large number of complete restorations in the deletion condition $(57 \%)$. The effect of context apparently overrode the fact that the initial segment of the target-bearing word was missing. Listeners were still able to perceive these nonwords as being intact.

However, in the distortion conditions, the acoustic information provided apparently conflicted with the context of the passage being shadowed. That is, the listeners heard the target-bearing word and realized that the target did not match any meaning appropriate to the context in which the target was embedded. Therefore, the listeners tended to repeat the word as it was spoken on the tape.

In light of the foregoing, it was apparent that subjects' perceptual strategies when shadowing words that were altered by distortion or deletion were quite different. When a subject was faced with deleted information, the missing phonetic information was not disruptive enough to disturb the fluent-speech-perception process, since the contextual constraints of the passage helped to fill in missing acoustic information in a somewhat "noisy" environment. However, when the acoustic information was distorted, the context was still important in helping to overcome some of the noise, but it was less effective in doing so, as seen by the large number of exact repetitions in the two distortion conditions.

Actually, the context may have been in conflict with acoustic information. A listener may have had a "mental set" of what was to come, based upon the context and the rhythm of an intended message. However, when conflicting auditory information began to be processed, the listener may have been led astray; he or she would then try to access words from the mental lexicon that fit both the misleading acoustic information (bottom-up) and the contextual information (top-down). Since subjects had a mismatch in information, they tended to respond with repetitions, since no such item existed in their mental lexicons. It is also possible that subjects tended to repeat misarticulations because the subject instructions for shadowing prompted repetitions.

\section{Feature Changes (Distortions)}

Although a sizable difference in number of subject restorations was seen between the cases of consonant distortion and consonant deletion, no significant difference was seen in relation to the number of restorations in the one-feature-change condition versus the three-featurechange condition; there was no difference between subjects' perceptions of one- versus three-distinctive-feature changes in word-initial phonemes. Although the number of restorations was greater for the one-feature-change con- 
dition than for the three-feature-change condition, the difference was not statistically significant. This is quite a disturbing state of affairs. At face value, it would be expected that a three-feature change would be more disruptive to lexical access than a one-feature change. A word would be more dissimilar to the original word if a larger number of features were changed, than if a smaller number of features were changed. The more dissimilar the target is to the intended word, the more difficult it should be to restore that word to its original form. Previous research has shown that as the number of feature changes increases, word recognition becomes more difficult (Cole, 1973; Marslen-Wilson \& Welsh, 1978).

One difference in results between previous research and the present study may be attributed to differences in the distinctive-feature classification systems employed. Both Cole (1973) and Marslen-Wilson and Welsh (1978) used the Keyser and Halle (1968) feature system, whereas in the present study the Chomsky and Halle (1968) system was employed. When examining differences between systems, we found some feature changes to be unequal. For instance, an $/ \mathrm{f} /$ to $/ \mathrm{s} /$ change is a three-feature change in the Chomsky and Halle system but only a two-feature change in the Keyser and Halle system.

A discrepancy in results between the Marslen-Wilson and Welsh (1978) study and the present study can be attributed to syllable position of the target. Marslen-Wilson and Welsh embedded mispronunciations in the third syllable of three-syllable words, whereas we placed all targets in the initial position of two-syllable words. Differences in number of subject restorations between words with syllableinitial and syllable-final mispronunciations can be predicted when viewed in the light of contextual constraints in word recognition (see Cole, Jakimik, \& Cooper, 1980).

\section{Deletions}

As for differences between words in the deletion condition of this study, it appears that the length of the deleted portion of each target had no apparent effect on listeners' restoration of the word. That is, for deletions ranging from 40.63 to $137.50 \mathrm{msec}$ (Table 3), listeners were able to completely restore the missing phonemic information $57 \%$ of the time (see Figure 1).

The percentage of restorations of deleted segments is much larger than the percentage of restorations in the distortion conditions. Why were deleted phonemes easier to restore? One explanation that could be put forward is that phonetic information was present in spite of the deletion attempts. This, however, is unlikely, since three experienced listeners transcribed the target items during construction of the experimental tape, and all three independently agreed that the target phonemes in all 14 target words were completely deleted.

Another possible explanation for such a high percentage of restorations is that subjects could not pronounce the words with deleted segments and that they therefore responded with restoration responses. However, if subjects could not pronounce the target words with deletions, they could have easily omitted the targets completely or constructed new words to fit the context of the passage. It is interesting that the subjects' omission rate in the deletion condition was only $11 \%$, which was consistent with deletions in the two distortion conditions. Likewise, subjects' meaningful new-word responses consisted of only $15 \%$ of the total response pool (see Figure 1).

Since these alternate explanations do not appear to account for the large number of subjects' restorations of deleted information, it appears that subjects can compensate for missing information. Salasoo and Pisoni (1985) have recently demonstrated that subjects were able to recognize words (in a modified gating paradigm) in which initial speech segments were replaced with envelopeshaped noise, in contrast to the complete speech-segment deletions used in the present study.

If the initial speech segment necessary for activation of a pool of word candidates is more on the magnitude of $150 \mathrm{msec}$, the deletion data in the present study can be explained in terms of cohort theory. Subjects in the present study had little difficulty in restoring words to their original form even with deletions as large as $137.5 \mathrm{msec}$. Apparently, subjects were still provided with enough acoustic-phonetic and contextual information so that word recognition could occur. This is in agreement with the findings of Salasoo and Pisoni (1985), who state that syntactic constraints appear to help guide the selection of words for the initial cohort while the first $150 \mathrm{msec}$ of acoustic-phonetic information is gathered by the listener. "At this point in time acoustic-phonetic information gains prominence while both top-down and bottom-up sources continue to eliminate incorrect candidates from the hypothesized set of words"' (p. 229).

In conclusion, when word-initial consonants were mispronounced, subjects had a difficult time in determining what the intended target should have been. However, when the initial phoneme was deleted, subjects tended to restore the deleted phonemic information. The simple deletion of a consonant was apparently not as disruptive to fluent speech perception as the misarticulation of a consonant. When a word-initial consonant was deleted, listeners were able to use contextual, lexical, and phonetic (as well as prosodic) information to fill in the missing phoneme, as would be done when listening in a poor acoustic environment. When the initial consonant was significantly distorted by changing its distinctive feature specifications, subjects tended to repeat the misarticulated phoneme, since the combination of context and conflicting acoustic-phonetic information could not be used to locate the item in their mental lexicons. In that case, subjects simply repeated the sequence of meaningless phonemes, indicating that when there was a significant alteration of a word-initial phoneme, a breakdown in the word-recognition process had occurred. 


\section{REFERENCES}

BAGLEY, W. C. (1900-1901). The apperception of the spoken sentence: A study in the psychology of language. American Journal of Psychology, 12, 80-130.

Bond, Z. S., \& SMALl, L. H. (1983). Voicing, vowel and stress mispronunciations in continuous speech. Perception \& Psychophysics, 34, 470-474.

Bruning, J. L., \& KINTZ, B. L. (1977). Computational handbook of statistics. Glenview, IL: Scott, Foresman.

Chomsky, N., \& HALle, M. (1968). The sound pattern of English. New York: Harper \& Row.

Clark, H. H. (1973). The language-as-fixed-effect fallacy: A critique of language statistics in psychological research. Journal of Verbal Learning \& Verbal Behavior, 12, 335-359.

Cole, R. A. (1973). Listening for mispronunciations: A measure of what we hear during speech. Perception \& Psychophysics, 13, 153-156.

Cole, R. A., \& JAKIMIK, J. (1980). A model of speech perception. In R. A. Cole (Ed.), Perception and production of fluent speech (pp. 133163). Hillsdale, NJ: Erlbaum.

COLE, R. A., JAKIMIK, J., \& CoOPER, W. E. (1980). Segmenting speech into words. Journal of the Acoustical Society of America, 67, 1323-1332.

KeYSER, S. J., \& HALLe, M. (1968). What we do when we speak. In
P. Kolers \& M. Eden (Eds.), Recognizing patterns. Cambridge: MIT Press.

KuČERA, F., \& Francis, W. N. (1967). Computational analysis of present day American English. Providence, RI: Brown University Press.

Liberman, A. M., Cooper, F. S., Shankweiler, D. P., \& StuddertKennedy, M. (1967). Perception of the speech code. Psychological Review, 74, 431-461.

Marslen-Wilson, W. D., \& Welsh, A. (1978). Processing interactions and lexical access during word recognition in continuous speech. Cognitive Psychology, 10, 29-63.

Peterson, G. E., \& Lehiste, I. (1960). Duration of syllable nuclei in English. Journal of the Acoustical Society of America, 32, 693-703.

Salasoo, A., \& PISONI, D. (1985). Interaction of knowledge sources in spoken word identification. Journal of Memory \& Language, 24, 210-231.

SAmUel, A. G. (1981). Phonemic restoration: Insights from a new methodology. Journal of Experimental Psychology, 110, 474-494.

TYLER, L. K. (1984). The structure of the initial cohort: Evidence from gating. Perception \& Psychophysics, 36, 417-427.

WARREN, R. M. (1970). Perceptual restoration of missing speech sounds. Science, 167, 392-393.

(Manuscript received September 26, 1985; revision accepted for publication May 10, 1986.) 